\title{
Surface EMG electrode distribution for Thumb Motion Classification Based on Wireless Communication Equipment
}

\section{Wanfen Xu, Gongfa Li*, Zhaojie Ju, Honghai Liu}

College of Machinery and Automation,

Wuhan University of Science and Technology, Wuhan, 430081, China

School of Computing, University of Portsmouth, Portsmouth PO1 3HE, UK

Email: one_fen@foxmail.com

Email:ligongfa@wust.edu.cn

Email:zhaojie.ju@port.ac.uk

Email: honghai.liu@port.ac.uk

*Corresponding author

\begin{abstract}
The interaction between humans and computers has become more necessary and more specific, and the informatization of human hands has made the human-machine interaction based on gesture recognition more and more extensive. Thumb as the most important finger plays a decisive role in decoding the gesture, especially in controlling of the smart phones and many other smart devices, etc. As a result, this study aims to decode the different thumb gestures from sEMG signal and to improve the robustness of gesture recognition and decrease the influence of physiological conditions and the electrode displacement between different users. In this article, we use the Bluetooth wireless communication and focus on the relationship between the EMG signal and the electrode identifier number. We change the electrode's number into a new feature and combining the traditional features with the new features to verify the electrode's number has a correlation with the thumb gesture. Experiments show that after adding new features, the gesture recognition rate has increased.
\end{abstract}

Keywords: EMG; Thumb; Gesture Recognition; wireless communication

Biographical notes: Wanfen Xu received her BS at Wuhan Polytechnic University. Her current research interests include mechanical $\mathrm{CAD} / \mathrm{CAE}$, signal analysis, and processing.

Gongfa Li received the Ph.D. degree in Wuhan University of Science and Technology, Wuhan, China. He is currently a professor in Wuhan University of Science and Technology. His major research interests are computer-aided engineering, mechanical $\mathrm{CAD} / \mathrm{CAE}$, modeling and optimal control of complex industrial process.

Zhaojie Ju received the B.S. in automatic control and the M.S. in intelligent robotics both from, Huazhong University of Science and Technology, China, in 2005 and 2007 respectively, and the $\mathrm{Ph} . \mathrm{D}$. degree, in intelligent robotics at the University of Portsmouth, UK, in 2010. Dr Ju is currently a Senior Lecturer, in the School of Computing, University of Portsmouth, UK. He previously held research appointments in, the Department of Computer Science, University College London and Intelligent Systems and Biomedical, Robotics group, University of Portsmouth, UK. His research interests are in machine intelligence, robot, learning and pattern recognition.

Honghai Liu received the Ph.D. degree in intelligent robotics from Kings College, University of London, London, U.K. He is currently a professor of School of Mechanical Engineering in Shanghai Jiao Tong University, Shanghai, China and a Professor of Intelligent Systems in Portsmouth University, Portsmouth, UK. His research interests are approximate computation, pattern recognition, multi-sensor based information fusion and analytics, human machine systems, advanced control, intelligent robotics and their practical applications.

\section{Introduction}

In the past ten years, many scholars have researched on gesture recognition, but there are not many researchers who focus on the thumb's movement. According to clinical statistics, more than $50 \%$ of the hand movements cannot be 
independent of the thumb, especially the grasping gesture. Above this, it can be inferred that the position of the thumb is very important when grabbing and the trajectory of the thumb can largely determine the quality of the grasping in hand. The muscles which control the thumb are divided into two groups, Intrinsic muscle, and Extrinsic Muscle. The Intrinsic muscle is distributed in the palms of the hand, and the Extrinsic Muscle is distributed in the forearms. Intrinsic muscle includes five muscles, which are Abductor Pollicis, Transversus (APT), Flexor Pollicis Brevis (FPB), Abductor Pollicis Brevis (APB), and First Dorsal Interosseus (FDI). And Extrinsic Muscle includes 4 muscles, namely Flexor Pollicis Longus (FPL), Abductor Pollicis Longus (APL), Extensor Pollicis Longus (EPL), and Extensor Pollicis Brevis (EPB) [1]. In contrast, the muscles on the palm are closer to the thumb, but its motility causes intrinsic muscles to be more prone to motion artifacts. And the extrinsic muscle is more fix than intrinsic muscle. it is convenient to distribution electrode. As showed in figure 1.

Figure 1 Extrinsic Muscle [2]

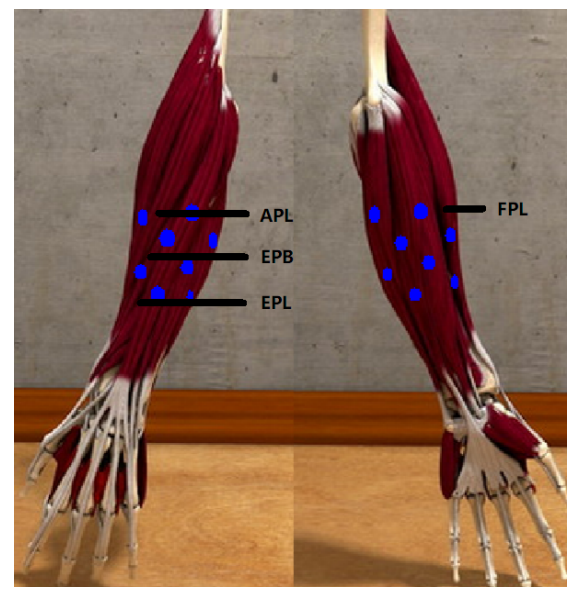

\section{Relate Work}

During the last decade, a large amount of researchers studied EMG signal based hand motion recognition, but most of them focused on the whole hand and few of them focused on the fingers. Xun Chen, and Z. Jane Wang have investigated the recognition accuracy affected by the features and the classification algorithms. The experiment used four channels to collect forearm sEMG signals from ten Chinese hand gestures. By the combination of traditional feature and the proposed classification algorithms, the hand movement recognition accuracy improved to more than 95\% [3]. Jongin Kim, Dongrae Cho, Kwang Jin Lee and Boreom Lee used sEMG signals to recognize the pinch-tozoom gesture by distance [4]. Nor Anija Jalaludin, Abu
Ubaidah Shamsudin, Shahrul Na'im Sidek et al designed a device to detect the sEMG and the force of the thumb to establish a sEMG model of the relation between thumb angle and force [5].

The current research focused on the electrode selection from 16 channels, and it aimed to find channels that are redundant for thumb motion classification across different subjects. In the meanwhile, it is also focused on the feature which is concerned about the identifier number of the electrode, finally to improve the classification rate.

\section{Experiment Design and Implementation}

\subsection{Experiment Design}

There are nine subjects in the experiment, seven males, and two females. All of them were about 25 years old and have no neural diseases. Subjects were asked to sit, and the hand relaxes hang in the air.

During the collection process, try to move only with the thumb and relax the other fingers. We collect the gesture takes the turn in 5 sessions which in order of up, down, left, right, press. Each session repeats the gesture 10 times. Each gesture lasts 10 seconds, 1 to 5 seconds is the relaxation phase, and 6 to 10 seconds is the gesture acquisition phase. It can be concluded that every gesture had collected 50 times, and there is total 2250 time of gesture in the database.

Figure 2 Thumb Gesture

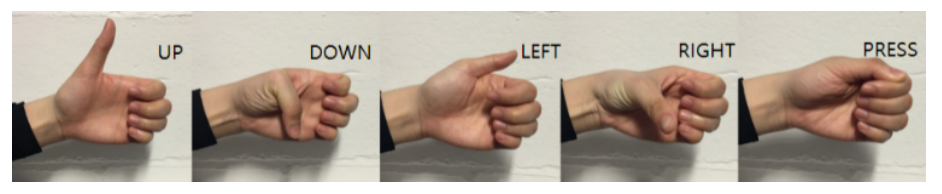

\subsection{Hardware selection}

EMG acquisition hardware is a 16-channel high temporal and spatial resolution sEMG collector with Bluetooth wireless communication and a sEMG sleeve with 18 dry electrodes. The EMG acquisition instrument is equipped with two main filters which are $50 \mathrm{~Hz}$ comb filters and 20 $\mathrm{Hz}$ high pass filters. It used to filter out power frequency noise and motion artifacts, respectively [6]. There are18 dry electrode on the sleeve which is uniformly distributed on the forearm, and each electrode has a corresponding number. Number 1 to number 16 is the channel number, there are two electrodes which are number ' 0 ', represent the reference electrode and the ground electrode, respectively [7].

Figure 3 Hardware in Experiment 


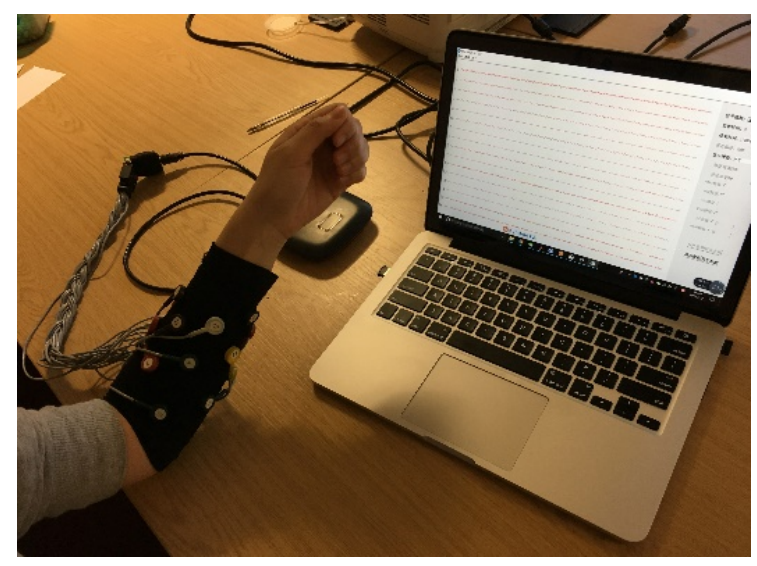

\subsection{Signal processing}

The raw signal from the acquisition device is a matrix which has 18 columns and 10000 rows. Column 1 to column 16 columns indicate the measured voltage values from 16 electrodes, and the column 17 to column 18 columns are used to verify Bluetooth module's transmission. The rows of the matrix are related to the acquisition time, that each row represents one millisecond. It follows that the original matrix is a very large matrix and we need to do dimensionality reduction.

\subsection{Dimensionality reduction}

\section{1) Active Segment}

Since each gesture consists of two parts which are relaxation and movement, and each part is 5 seconds. We remove the first 5 seconds of each gesture and keep the movement part. For ensuring the integrity of the whole motion signal, we set the start point at 4.8 second of each gesture.

\section{2) Delete Redundant Channels}

Not all muscles located in the forearm are associated with the thumb. It leads some of the electrodes are redundancy. We need to delete these redundant channels, thereby increasing the speed of calculating [8].

$$
V A R=\frac{1}{N-1} \sum_{i=1}^{N} x_{i}^{2}
$$

We removed one subject respectively and accumulated the remaining eight subjects' coefficients by channel. Every channel has their own corresponding coefficient value. It can be assumed that the channel with the larger value is the more redundant, whereas the channel with the smaller value is the more useful [9].

Arrange the value into 16 levels in order. Separate evaluating the channel values for each subject set and assign the level label. The label in this experiment is 16 different colors. Then choose their common redundant channel as the redundant channel. Depending on Figure 4, the most redundant channel is number 13 .
Figure 4 Most Redundant 10 Channels of Each Subject

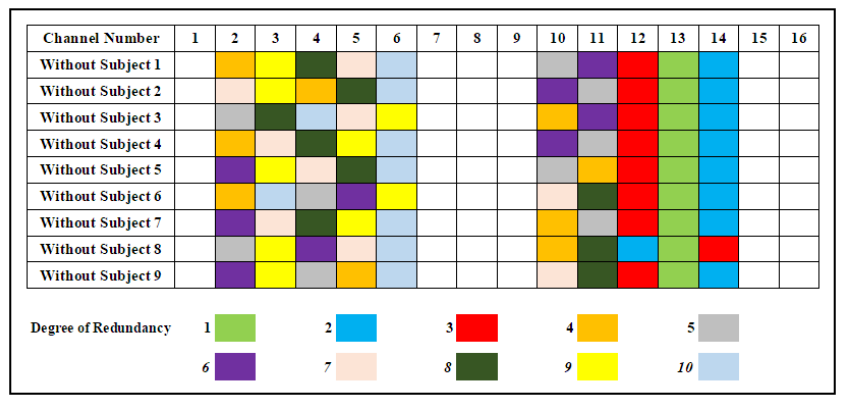

Figure 4 shows that for the different subject, there are three same redundancy channels which are No. 13, No. 14 and No. 12 , respectively, and the most ten redundant channels are number 2 to number 6 and number 10 to number 14 .

According to the above figures, there are three types of common redundant channels which are removing one channel, removing three channels, and removing ten channels [10]. According to tests, removing one redundant channel and removing three redundant channels has no effect on the classification result. When removing 10 redundant channels, the classification effect is significantly decreased. In concluding, we chose to remove three redundant channels.

\subsection{Feature Extraction}

The experiment combines traditional features with new features. The traditional features use four kinds of timefrequency characteristics, which are the number of slope sign changes feature (SSC), Window Length feature (WL), The number of zero crossings feature (ZC) and the Mean Absolute Value features(MAV).

The new feature is based on the MAV feature matrix, and the matrix converts the MAV voltage value to the corresponding color. Each element in the matrix corresponds to a color match the color blocks form a rectangular color map. We intuitively find the relationship between the electrode and muscle activity from the figure, thus classifying the gestures.

For each time of gesture, the strength is different, and it will result in a different intensity of the EMG signal. Therefore, the MAV matrix needs to be normalized. Dividing each element of the matrix by the maximum value in the matrix. After processing the MAV matrix, the maximum value of the element is 1 .

\subsection{Matrix Interpolation}

Since we chose a 16-channel sleeve, it leads the MAV matrix has only 16-rows. In the 16-rows MAV matrix, the color block is large, it makes the graphics formed by each gesture are unclear. We do an interpolation operation on this matrix, which can improve the resolution of the graphics, and will not increase the computing [11].

Figure $5 \mathrm{MAV}$-features matrix of the UP gesture 


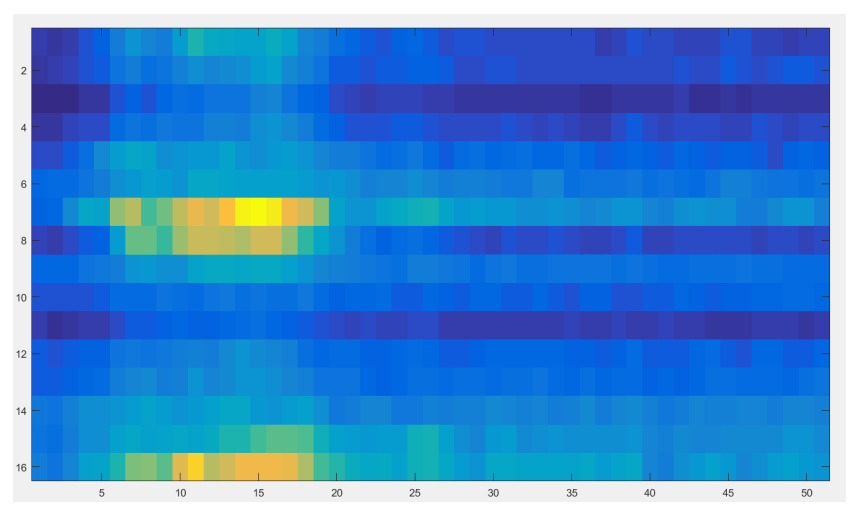

Figure 6 Matrix after interpolation of the UP gesture

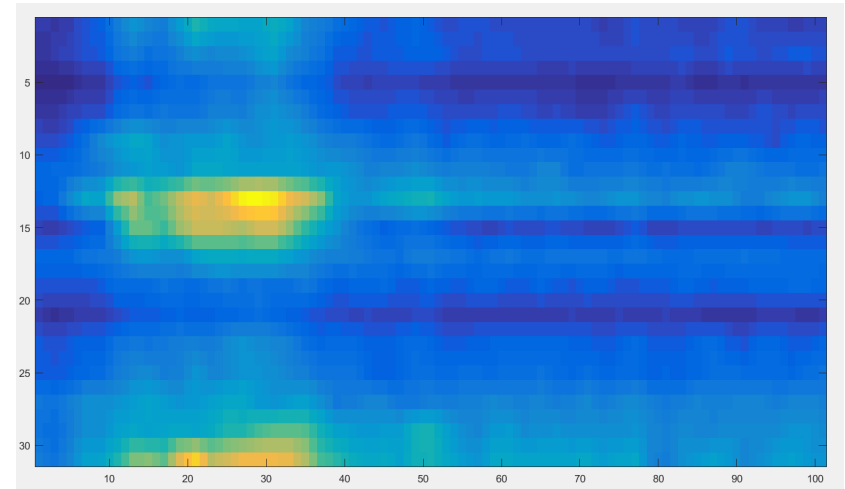

3.7 Edge extraction

The contour lines characterized the signal distribution in the same intensity from all intensities. Using contour lines, find the corresponding graph for each value from 0 to 1 . The test shows that when the layer height is 0.4 , the amount of information in the graph is the largest.

Figure 7 the UP gestures at different heights

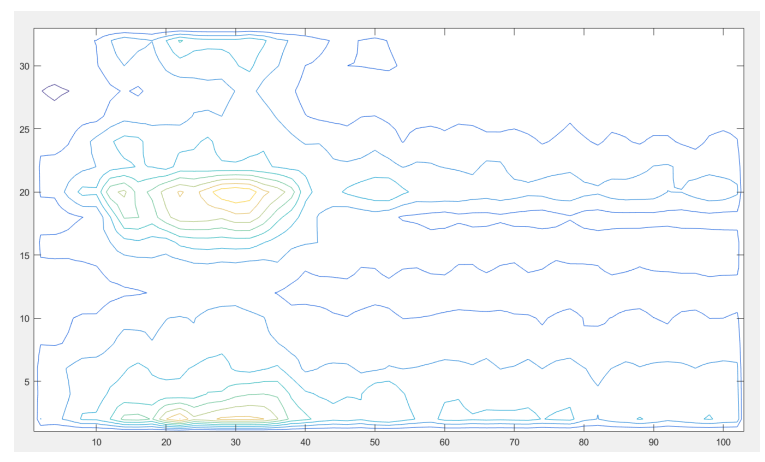

For the different subject, they will generate the different EMG signal when making the same gesture in different times, but the muscle which is relative motion is consistent. It can be inferred that the relatively active electrodes in it have a certain correlation with the gesture. In the meanwhile, there is a certain correlation between the outline information and the gesture [12].
We make a rectangle tangent to the outline and take the ordinate of the tangent as the feature element [13]. Similarly, we also extract the other element as the feature elements, such as the geometric center of gravity of the contour, the coordinates of the extreme points in the image, the coordinates of the redundant channel sitting, etc.

Figure 8 the UP gestures at the height of 0.4

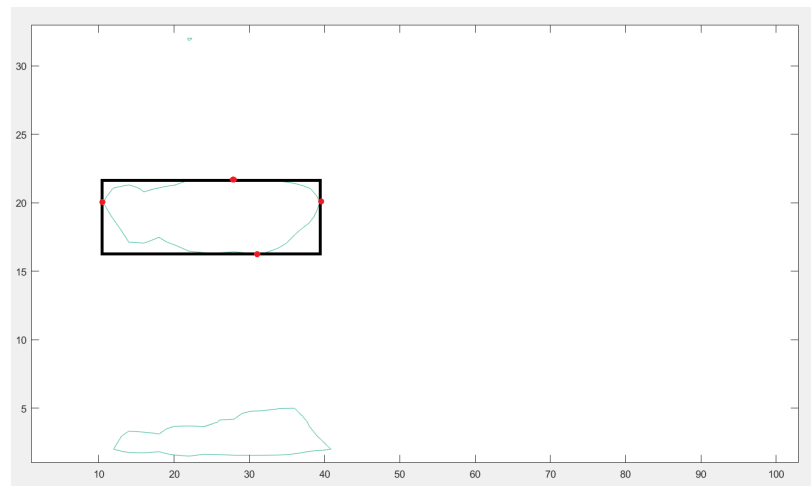

\section{Evaluation}

We use the intra-session method to train $9 / 10$ of the data in each session and test the remaining 1/10 of the data. Test results as showed in figure 9 . After adding the new features, the classification effect of some groups has been improved to some extent, while other groups have not changed. The total recognition rate has increased. The final recognition rate can reach $94.2223 \%$.

Figure 9 the recognition rate of traditional features and new features

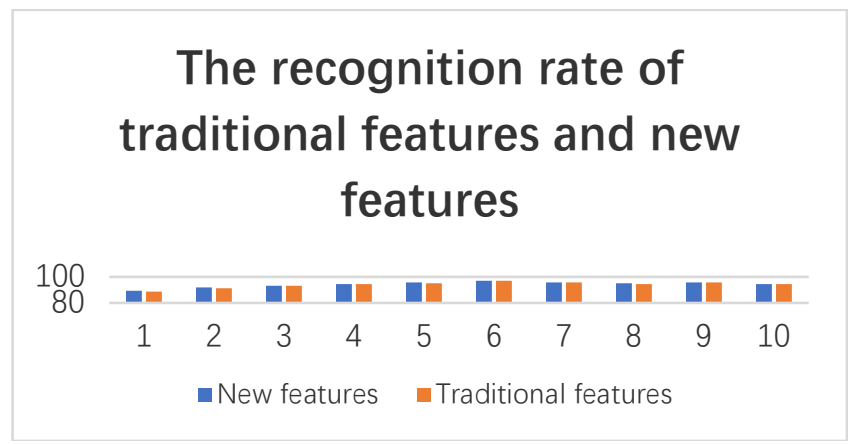

There were total 9 subjects in the experiment. For each subject, there was divided into five sessions. Every session is divided into five gestures, and each gesture repeated ten times, total 2250 time of gestures in the database. We counted the results of data which classified by the SVM method and made a table of the results of each gesture prediction. The horizontal row is the data which actual test gesture, and the vertical row is the data which predicted by SVM method.

Figure 10 SVM Classification Results Statistics 


\begin{tabular}{|c|c|c|c|c|c|}
\hline & UP & DOWN & LEFT & RIGHT & PRESS \\
\hline UP & 414 & 0 & 34 & 2 & 0 \\
\hline DOWM & 3 & 426 & 2 & 15 & 4 \\
\hline LEFT & 33 & 2 & 413 & 2 & 0 \\
\hline RIGHT & 12 & 4 & 3 & 428 & 3 \\
\hline PRESS & 0 & 4 & 3 & 4 & 439 \\
\hline
\end{tabular}

Jongin Kim 1, Dongrae Cho 2, Kwang Jin Lee et al. A Real-Time Pinch-to-Zoom Motion Detection by Means of a Surface EMG-Based Human-Computer Interface[J]. Sensors, 2015, 15, pp.394-407

Nor Anija Jalaludin, Abu Ubaidah Shamsudin, Shahrul Na'im Sidek et al. Electromyography (EMG)-based Thump-tip Force Estimation for Prosthetic Thumb[C]. International Conference on Computer and Communication Engineering, 2012

Abdul Rahman Siddiqi, Shahrul Nairn Sidek, Muhammad Rozaidi Roslan. EMG based Classification for Continuous Thumb Angle and Force Prediction[J]. International Symposium on Robotics and Intelligent Sensors, 2015, pp. 43-47

A. Aranceta-Garza, H. Lakany, B. A. Conway. An investigation into thumb rotation using high density surface electromyography of extrinsic hand muscles[C]. International Conference on Systems, International Conference on Systems, 2013

\section{Result}

The new feature is the contour ordinate coefficient of the MAV matrix transformation, and the dimensions of the new feature are much smaller than the dimensions of the traditional feature. There is a certain mapping relationship between the ordinate coefficient and the electrode identifier number. Although different samples wear sleeves will produce different degrees of displacement phenomenon, and each sample to do the same gesture is also different [14]. The new features are used in the process of gesture recognition to increase the recognition rate. This shows that there is a certain link between the thumb gesture and the electrode identifier number.

\section{Discussion and conclusion}

In this experiment, we had confirmed the position where the forearm did not produce the electrical signal of the thumb by recognition of the movement of the thumb, and we also confirmed the number of the electrode has a certain correlation with the thumb's gesture [15]. But the sleeve used in this experiment had only 16 channels, the limitation of the electrodes resulted in redundant locations and active locations were not specific enough. It is obvious that further research will focus on high-density electrode sleeve to find more redundant areas locations and more active areas locations in the forearm, further to invent the highprecision and low-cost sEMG device.

\section{References}

Abdul Rahman Siddiqi, Shahrul Naim Sidek, Aida Khorshidtalab. Signal Processing of EMG Signal for Continuous ThumbAngle Estimation[J]. IECON,2015, pp. 374-379.

Yinfeng Fang, Zhaojie Ju, Xiangyang Zhu et al. Finger Pinch Force Estimation Through Muscle Activations Using A Surface EMG Sleeve On the Forearm[C]. IEEE International Conference on Fuzzy Systems, International Conference on Fuzzy System, 2014

Muhammad Rozaidi Roslan, Shahrul Naim Sidek, Siti Fauziah Toha et al. Portable Thumb Training System for EMG Signal Measurement and Analysis[C]. International Conference on Computer \& Communication Engineering, 978-1-5090-2427$8 / 16,2016$

Xun Chen, Z. Jane Wang. Pattern recognition of number gestures based on a wireless surface EMG system[J]. Biomedical Signal Processing and Control, 2013, pp. 184-192

Y. Fang, X. Zhu, and H. Liu. Development of a surface emg acquisition system with novel electrodes configuration and signal representation[C]. Lecture Notes in Computer Science, 2013, vol. 8102, pp. 405-414.

Jongin Kim, Dongrae Cho, Kwang Jin Lee et al. A Real-Time Pinch-to-Zoom Motion Detection by Means of a Surface EMG-Based Human-Computer Interface[J]. Sensors, 2015, vol. 15, pp. 394-407

L R Quitadamo, F Cavrini, L Sbernini et al. Support vector machines to detect physiological patterns for EEG and EMGbased human-computer interaction: a review[J]. Journal of Neural Engineering,2017, pp. 14:1-27

Abdul Rahman Siddiqi, Shahrul Naim Sidek. Estimation of continuous thumb angle and force using electromyogram classification[J]. International Journal of Advanced Robotic Systems, 2016, pp. 1-12

Visakha K. Nanayakkara, Giuseppe Cotugno, Nikolaos Vitzilaios et al. The Role of Morphology of the Thumb in Anthropomorphic Grasping: A Review[J]. Frontiers in Mechanical Engineering, 2017, vol. 3, no.5, pp. 1-21

Nor Anija Jalaludin, Abu Ubaidah Shamsudin, Shahrul Na'im Sidek et al. Electromyography (EMG)-based Thump-tip Force Estimation for Prosthetic Thumb[J]. International Conference on Computer and Communication Engineering, 2012, pp. 3-5

Yinfeng. Fang, Honghai. Liu, Gongfa. Li et al. A Multichannel Surface EMG System for Hand Motion Recognition[J]. International Journal of Humanoid Robotics, 2015,12 